\title{
Enunciación
}

http://revistas.udistrital.edu.co/ojs/index.php/enunc

DOI: http://doi.org/10.14483/22486798.11544

REPORTE DE CASO

\section{Crisis de las humanidades y relación ser humano/naturaleza: una experiencia en la formación de maestros de lengua y literatura}

\author{
Crisis of the Humanities and the Relationship between the Human Being and \\ Nature: An Experience in Training Teachers
}

\author{
Carlos Andrés Parra Mosquera*
}

\section{Resumen}

Este artículo se deriva de una propuesta de práctica profesional de la Licenciatura en Educación Básica con énfasis en Humanidades, Lengua Castellana, de la Universidad de Antioquia, implementada durante 2016 y 2017-I con estudiantes de últimos semestres. Esta propuesta ha sido inédita y precursora en dicho programa, pues pretende favorecer la problematización y la resignificación de la relación entre el ser humano y la naturaleza, en el ámbito de la formación en lenguaje, a partir de la dimensión estética y de diversos desafíos disciplinares, inter- y transdisciplinares que se sitúan en la actual crisis de las humanidades. En un primer momento, se presentan elementos generales de la propuesta y el panorama donde ella se posiciona. El segundo momento describe cuatro ejes centrales de la propuesta. El primero aborda la pertinencia e implicaciones de incorporar la relación del ser humano con la naturaleza en un pregrado disciplinar orientado a la enseñanza de la lengua castellana y la literatura en la escuela. El segundo remite al desafío y la oportunidad que implica emprender una experiencia más allá de los límites disciplinares. El tercero se refiere a la formación de maestros en contextos no escolares. El cuarto alude a cuestiones emergentes, en cuanto a la formación en investigación y el diálogo con saberes indígenas. Finalmente, se presentan algunas conclusiones del proceso.

Palabras clave: formación de profesores, humanidades, educación ambiental, conocimientos tradicionales, educación alternativa.

\begin{abstract}
This article presents the pedagogical practice proposal of the Bachelor in Basic Education with emphasis in Humanities and Spanish Language, at the Faculty of Education at the University of Antioquia. The proposal has been implemented during 2016/2017-I with students who are developing their undergraduated project. The proposal is pioneering in redefining the relationship between humans and nature in the field of literacy, from an aesthetic dimension. It implies several disciplinary challenges, addressed by the inter and transdisciplinary perspectives. In the introduction the article presents an overview of the proposal. Then, it focuses on four axes of the pedagogical practice proposal: first it deals with the relevance and implications of incorporating the relationship of humans with nature, in the field of a discipline traditionally oriented to the acquisition of language and literature in school. Second, it refers to the opportunity that comes with crossing disciplinary boundaries. Third, it relates the training of teachers to non-school contexts. To conclude, the article brings the emerging trend in research that dialogues with indigenous knowledge, as a key to train new generations of undergraduate students. Finally, it presents some conclusions.
\end{abstract}

Keywords: teacher training, human studies, environmental education, traditional knowledge, alternative education

Doctor en Ciencias Sociales y Humanas, Pontificia Universidad Javeriana-Bogotá; magíster en Educación y licenciado en Educación Básica con énfasis en Humanidades, Lengua Castellana, Universidad de Antioquia. Profesor de cátedra y asesor de práctica profesional, Universidad de Antioquia. Correo electrónico: cadrep@ gmail.com; andres.parra@udea.edu.co.

Cómo citar este artículo: Parra Mosquera, C.A. (2017). Crisis de las humanidades y relación ser humano-naturaleza: una experiencia en la formación de maestros de lengua y literatura. Enunciación, 22(2), 217-230. DOI: http://doi.org/10.14483/22486798.11544

Artículo recibido: 29 de enero de 2017; aprobado: 17 de octubre de 2017. 


\section{Coordenadas y alternativas de una propuesta de práctica profesional}

La propuesta de práctica profesional intitulada "In-disciplinar la formación en lenguaje desde otros sistemas simbólicos (no verbales): elaboración e implementación crítica de materiales didácticos sobre la relación del ser humano con la naturaleza en contextos no escolares", tuvo lugar en la Licenciatura en Educación Básica con énfasis en Humanidades, Lengua Castellana, de la Universidad de Antioquia. Allí, la práctica profesional se asume

[...] como un escenario donde los maestros en formación se posicionan como sujetos de saber y como sujetos políticos capaces de aportar, desde su sensibilidad, sus apropiaciones, sus discursos y sus procesos investigativos, a las prácticas de enseñanza en el campo de lenguaje que tienen lugar en la escuela o en otros espacios educativos. (Licenciatura en Educación Básica con énfasis en Humanidades, Lengua Castellana, 2016, p. 3)

Desde tal marco, los objetivos generales de la propuesta que da lugar a este texto fueron:

Problematizar la relación entre el ser humano y la naturaleza, a partir de la dimensión estética en el ámbito de la formación en lenguaje y desde una apuesta investigativa colaborativa que explore los desafíos inter- y transdisciplinarios.

Bosquejar una propuesta de mediaciones y otros sistemas simbólicos derivada de la lectura crítica de contextos educativos no escolares y a partir de discusiones y experiencias tendientes al análisis, diseño, implementación y construcción colaborativa de materiales didácticos que favorezcan nuevas percepciones y comprensiones de la relación entre ser el humano y la naturaleza frente a las miradas predominantes en la actualidad. (Parra, 2016a, p. 3)

Si bien no es el énfasis de este texto, vale la pena describir, además, las apuestas metodológicas centrales que le dieron vida a todo el proceso.
Durante tres semestres (2016 y 2017-I), los estudiantes participaron de dos espacios formativos. El primero, situado en la universidad; donde los encuentros presenciales conjugaban elementos del seminario y del taller para favorecer discusiones conceptuales y experiencias de trabajo colaborativo que involucraban sentidos del conocimiento, no solo centrados en componentes teóricos, lógicos y declarativos. El segundo fue el centro de práctica, es decir, la Corporación de Educación Ambiental GEDI; allí se acentuaba el componente colaborativo de diversas maneras, como fueron los conversatorios, debates, propuestas formativas grupales, elaboración de materiales didácticos, ejercicios argumentativos y registros del proceso. Esto fue vital para favorecer la negociación y resolución de los conflictos propios de contextos educativos no escolares, como se detalla luego. Mediante las estrategias descritas, se configuraron los proyectos de investigación y propuestas didácticas de los estudiantes, a través de un posicionamiento discursivo (conceptos, referentes, autores y experiencias) y estratégico (métodos, técnicas y recursos heurísticos e investigativos en espacios de intercambio y construcción presencial y virtual) reflejados en sus trabajos finales ${ }^{1}$. Al final, los resultados para cada semestre fueron:

Semestre 1: Configuración del proyecto de investigación y propuesta de mediaciones y otros sistemas simbólicos en cuanto a la formación sobre la relación entre el ser humano y la naturaleza en contextos no escolares.

1 Los trabajos de grado resultantes de este proceso pueden consultarse en el repositorio de la Facultad (http://ayura.udea.edu. co:8080/jspui/). Por eso, aquí referenció únicamente sus títulos y los nombres de sus autores: la alimentación desde lo afrochocoano como práctica decolonizadora del lenguaje: retos y aproximaciones a partir de una metodología decolonial (Vanessa Ospino y Karla Rodríguez); cuerpo, muerte y formación integral: escenarios de experiencias, conversaciones y significaciones a partir de procesos educativos en relación con la formación integral en lenguaje (Stefany Sepúlveda y Crystian Tobón), y formación en lenguaje a través de la alimentación pensada como un sistema simbólico (Elizabeth Duque). 
Semestre 2: Socialización de avances acerca de las experiencias e implementación de materiales didácticos consistentes con cada propuesta y su apuesta inter-transdisciplinaria para contextos no escolares.

Semestre 3: Entrega del texto escrito y sustentación oral ante la comunidad académica de la Facultad y del centro de práctica para dar cuenta del proceso formativo e investigativo de los estudiantes. (Parra, 2016a, p. 4)

Dado que esta ha sido una experiencia inédita para la Licenciatura, el énfasis y aporte de este texto está en los ejes conceptuales y tensiones epistémicas que emergieron durante el proceso de diseño e implementación de la propuesta de práctica profesional. Decidimos privilegiar este componente porque ha ofrecido herramientas y argumentos para afianzar las apuestas y exploraciones de la Licenciatura por construir otras lecturas y posibilidades de las humanidades en la formación de maestros del área del lenguaje. Y quizá, pueda resultar de utilidad para iniciativas afines que surjan en otras universidades.

Las coordenadas generales que inspiraron, justificaron y orientaron la propuesta de práctica profesional se sitúan en la crisis de las humanidades, contemplada desde una perspectiva histórica y no disciplinar. Al respecto, cabe destacar el modo en que, recientemente, ha cobrado relevancia mediática la cuestión de las humanidades en las universidades a través de artículos de prensa, comunicados de profesores universitarios y eventos a nivel nacional e internacional. Un ejemplo de la crisis de las humanidades es que "a partir de la década de los 90, [...] la concepción de 'conocimiento' que maneja Colciencias parece ser tributaria directa de las ciencias formalizadas, con lo cual otras formas de producir conocimientos encuentran dificultad para inscribirse a estas políticas (Castro-Gómez, 2014, p. 1).

Por otra parte, Marta Nussbaum (2010) y Nuccio Ordine (2013) son dos representantes insignes de la lectura predominante de esta problemática, denominada crisis de las humanidades. En su lectura prevalece una respuesta que defiende el lugar y capital simbólico, tanto de las artes como de las humanidades, en instituciones escolares, científicas y académicas. Resulta útil referenciar dos planteamientos medulares de esta postura porque permiten, de un lado, tomar distancia de su carácter dicotómico, disciplinar y, en cierta medida, ahistórico, y, de otro lado, ubicar los ejes y tensiones que acompañaron la propuesta de práctica profesional.

Nussbaum se refiere a la crisis de las humanidades como expresión de una crisis educativa y social.

[...] se están descartando sin advertirlo ciertas aptitudes que son necesarias para mantener viva a la democracia. [...] se están erradicando materias y carreras relacionadas con las artes y las humanidades (en todos los niveles educativos) en un momento en que las naciones deben eliminar todo lo que no tenga ninguna utilidad para ser competitivas en el mercado global. (2010, p. 20)

Es oportuno destacar dos rasgos de esta lectura y apología de las humanidades que ha calado tanto en diversos sectores de la academia y, particularmente, en la formación de maestros de Humanidades, Lengua Castellana. El primer rasgo es su carácter dicotómico. Resulta evidente un sentido reivindicativo del arte y de las humanidades canónicas, desde una postura que discrimina entre alta y baja cultura y en franca separación de las ciencias básicas y aplicadas. Tal aproximación desconoce la jerarquía epistémica que instaura el "modelo disciplinario de conocimiento que se consolidó como hegemónico durante los siglos XIX y XX" (Castro-Gómez, 2013, p. 37). Por tanto, naturaliza la compartimentación de los saberes disciplinares y su relación con el modelo cartesiano, herencia y rasgo vivo de la diferencia colonial; en palabras de Castro-Gómez, esto disminuye las posibilidades de cuestionar "la rigidez y la compartimentalización de esos saberes, así como su sospechosa complicidad con los poderes dominantes" (2013, p. 38). 
Adicionalmente, al acentuar la escisión entre las humanidades, las ciencias sociales y las ciencias básicas y aplicadas, se limitan las posibilidades de elaborar propuestas y alternativas, pues "la estructura arborescente de la universidad contribuye a separar los conocimientos, a disociarlos, a 'babelizarlos', e impide los diálogos interdisciplinarios y los devenires transdisciplinarios" (p. 38).

El segundo rasgo de la lectura en auge de la crisis de las humanidades remite a su inquietante desconocimiento o invisibilización de procesos históricos y contextuales. Al respecto, reconocemos una llamativa resonancia entre el clamor contemporáneo de Ordine (2013) con lo planteado por Alberto Urdaneta, gestor y fundador de la Escuela de Bellas Artes de la Universidad Nacional de Colombia en 1886. Ordine (2013) recalca, con cierta alarma, que la filosofía, la poesía, la historia y los autores clásicos son saberes que nos hacen más humanos e imprescindibles para nuestras democracias y para crear ciudadanos conscientes, comprometidos y mejores personas. Urdaneta sostenía que "la propagación de las artes y su perfeccionamiento son señales de la civilización de un país; son el límite que separa la cultura de la barbarie, y lo que diferencia del hombre inculto al inspirado artista" (citado por Castro-Gómez, 2014, pp. 9-10).

Resulta útil resaltar una continuidad entre Ordine y Urdaneta asociada al dispositivo escolar moderno, en el cual las humanidades han sido una herramienta de consolidación de los Estados-nación, mediante la unificación y homogenización. Esto lo explica Castro-Gómez, con base en el trabajo de Beatriz González sobre los dispositivos disciplinarios de poder en el contexto latinoamericano del siglo XIX:

[...] si la constitución define formalmente un tipo deseable de subjetividad moderna, la pedagogía es el gran artífice de su materialización. Lo que se busca es introyectar una disciplina sobre la mente y el cuerpo que capacite a la persona para ser "útil a la patria" [...]. No se escribieron manuales para ser buen campesino, buen indio, buen negro o buen gaucho, ya que todos estos tipos humanos eran vistos como pertenecientes al ámbito de la barbarie. (Castro-Gómez, 2000, p. 3)

A diferencia de Nussbaum y Ordine, la crisis de las humanidades, en nuestra propuesta de práctica profesional, se abordó desde una perspectiva histórica en relación con el proyecto modernidad-colonialidad (Escobar, 2016). Así hemos evitado dos cosas. Primero, reforzar un sentido predominante de las humanidades "que perpetúa la idea de que existen obras de arte intemporales que abordan los grandes problemas de la humanidad eterna a través de algunos lugares comunes ideológicos. Al hacerlo, enmascara la realidad social e histórica de los conflictos, los sufrimientos o los destinos singulares" (Chartier, 2004, p. 68). Segundo, evitamos limitar la crisis de las humanidades a disputas dicotómicas y maniqueas por contenidos curriculares disciplinares que desconocen e invisibilizan un horizonte histórico complejo de relaciones de saber/poder que se condensa en dinámicas institucionales y prácticas cotidianas. Si bien ambas situaciones se han sorteado, al menos por escrito, en el documento de transformación curricular de la Licenciatura en Humanidades, Lengua Castellana, de la Universidad de Antioquia, aún predominan los sentidos dicotómicos, ahistóricos y disciplinares en las aulas, las reuniones de profesores y en las prácticas cotidianas.

En relación con los desafíos y las alternativas frente a la crisis de las humanidades, conviene ir a De Sousa. Él sugiere que nos hallamos en un proceso complejo de transición del paradigma de la modernidad hacia un ámbito de incertidumbre que, potencialmente, puede dar lugar a la emergencia de otro(s) paradigma(s) y alternativas.

[...] estamos entrando en una fase de crisis paradigmática y, por lo tanto, de transición entre paradigmas epistemológicos, sociales, políticos y culturales. Se asume también que no basta continuar criticando el paradigma aún dominante, lo que por lo demás se ha hecho ya hasta la saciedad. Es necesario, además, definir el paradigma emergente. Esta última tarea que de lejos es la más importante, también es, de lejos, la más difícil. (De Sousa, 2012, p. 423) 
Según esto, no basta con los diagnósticos y críticas repetitivas sobre documentos de transformación curricular, pues la definición del paradigma emergente sucede en el ámbito de las prácticas y en tensión con la forma de conocimiento hegemónico de la modernidad, pues

[...] no hay una forma única de conocimiento válido. Hay muchas formas de conocimiento, tantas cuantas sean las prácticas sociales que las generan y las sostienen [...]. Prácticas sociales alternativas generan formas alternativas del conocimiento. El genocidio que puntualizó tantas veces la expansión europea, fue también un epistemicidio: se eliminaron pueblos extraños porque tenían formas de conocimiento extraño y se eliminaron formas de conocimiento extrañas porque se basaban en prácticas sociales y en pueblos extraños. (pp. 430-431)

El vínculo indisoluble entre la forma del conocimiento hegemónico de la modernidad y el epistemicidio ofrece una perspectiva de la crisis de las humanidades diferente a la lectura de Nussbaum y Ordine. En América, la jerarquización étnica y moral se trasladó al ámbito de la epistemología (Castro-Gómez, 2005). Las formas de conocimiento y el lenguaje asociados a la racionalidad técnico-científica formaron parte de una estrategia epistémica de los diseños coloniales para construir "una jerarquía de conocimientos superiores versus conocimientos inferiores $y$, por lo tanto, de seres superiores versus seres inferiores en el mundo" (Grosfoguel, 2006, p. 152). Allí encontramos, para nuestra propuesta de práctica, una coordenada relevante para sustentar la exploración de saberes no hegemónicos (indígenas y populares) y lugares no académicos de producción de saber (corporaciones y movimientos sociales).

Ahora bien, explorar las prácticas sociales alternativas sugeridas por De Sousa es una invitación que implica retornar a lo local. Lo cual consiste en rastrear y potenciar "cualquier condición, experimento, iniciativa o concepción política y social suprimida con éxito por las formas hegemónicas de la globalización, o aquellas que no se ha permitido que existan ni sean pronunciables como necesidad o aspiración" (2009, p. 233). Según lo anterior, en nuestra propuesta consideramos plausible la incorporación de saberes inéditos (o una resignificación de los disciplinares) en la escuela y en la formación de maestros. Saberes no oficiales, ni legitimados institucionalmente, que remiten, por ejemplo, a las emociones, la corporeidad, lo afro, lo indígena. Saberes que, en suma, han sido despreciados, invisibilizados e instrumentalizados, en la academia y en la escuela, por las miradas dominantes de las humanidades.

Tal apuesta derivó en una tensión de interés que, en palabras de Saldarriaga (2003), forma parte de los conflictos contemporáneos de la escuela y de la formación de maestros.

En la dimensión cultural, hasta hace poco excluida de los análisis pedagógicos [...] la tensión que aparece puede expresarse diciendo que si la función clásica de la escuela ha sido la de unificar las diferentes matrices culturales populares bajo la matriz unidireccional de la cultura ilustrada, tecno-científica, racional y letrada, ¿cuál es el umbral mínimo de permeabilidad de la escuela a las subjetividades juveniles locales y globales, a las identidades colectivas de los diversos grupos sociales, a las matrices estéticas y éticas provenientes de la multiculturalidad y la etnodiversidad, en un país de la periferia occidental y en tiempos de globalización económica y mundialización cultural? (pp. 144-145)

Por lo anterior, a nivel educativo, la crisis de las humanidades no debe reducirse a una disputa en el papel por los linderos y contenidos curriculares. Esto va más allá de una simple sustitución o adición de nuevos temas, pues las lógicas no clásicas de otros saberes requerirían de la apertura a otras miradas del mundo. Por ello, la escolarización de nuevos saberes, así como la resignificación y la experimentación de los saberes disciplinares corresponden a procesos que interpelan las formas del conocimiento hegemónico. Por ende, este proceso no consiste en implementar reformas curriculares que, seguramente, harían eco del rasgo de la racionalidad científica hegemónica de la modernidad 
que consiste "en la transformación de los problemas éticos y políticos en problemas técnicos" (De Sousa, 2012, p. 422). Máxime cuando se trata de una transición de paradigmas, que según Capra, "requiere una expansión no solo de nuestras percepciones y modos de pensar, sino también de nuestros valores" (2003, p. 31).

Insistimos en que el panorama presentado hasta ahora forma parte de algunos de los aprendizajes, argumentos y herramientas derivados de la propuesta de práctica profesional que han permitido dialogar con los pares académicos (profesores, estudiantes y administradores) de la Licenciatura para encuadrar, justificar y lograr su continuidad. En la misma perspectiva se ubican los ejes que se presentan a continuación.

\section{Ejes emergentes durante el proceso}

Desde la elaboración de la propuesta hasta su implementación, emergieron cuatro ejes desde donde interpelar la estructura disciplinar y dicotómica que predomina en el ámbito de las prácticas profesionales de la Licenciatura.

\section{Lo ambiental, la naturaleza y lo estético}

El primer eje apuntó a explorar las motivaciones y las posibles implicaciones de la incorporación de la relación del ser humano con la naturaleza en los intereses y agenda de un pregrado cuyas raíces y límites disciplinares se dirigen a la enseñanza de la lengua castellana y la literatura en la escuela regular. Este eje tuvo una vinculación directa con la crisis de las humanidades asumida en relación con la modernidad, como se presentó anteriormente. Según Castro-Gómez, el proyecto de la modernidad, "demandaba, a nivel conceptual, elevar al hombre al rango de principio ordenador de todas las cosas [...] viene de la mano con la idea del dominio sobre la naturaleza mediante la ciencia y la técnica" (2000, p. 2). En el mismo sentido, Ángel Maya afirmaba, "las ciencias naturales quieren explicar una naturaleza sin hombre, las ciencias sociales prefieren un hombre sin naturaleza" (como es citado en Ministerio del Medio Ambiente-Ministerio de Educación, 2002, p. 30). En efecto, las aproximaciones disciplinares al conocimiento que han predominado, tanto en la escuela como en la formación de maestros, han sido herederas y beneficiarias de esta dicotomía.

Por otro lado, múltiples estudios reconocen una crisis en la relación entre el ser humano y la naturaleza que se vincula con aspectos sociales, políticos, culturales, económicos, tecnológicos y educativos (Morín, 2011, p. 80). En Colombia, esta problemática ha hecho parte de las políticas públicas nacionales. La más reciente iniciativa en este campo se dio en el año 2002², cuando se promulgó la Política Nacional de Educación Ambiental. En su línea de base se reconocen

[...] pocos resultados en las acciones que ha emprendido la Universidad para incorporar la dimensión ambiental, desde la transversalidad de la temática, en sus procesos de formación, investigación y extensión, y particularmente en aquellos relacionados con los procesos de formación de docente. (Ministerio del Medio Ambiente-Ministerio de Educación, 2002, p. 12)

Ante lo cual se presenta como reto y estrategia central, "incluir la dimensión ambiental en los currículos de los diferentes programas que ofrecen las universidades, con especial énfasis en aquellos que tienen que ver con formación inicial de docentes" (p. 56). Pero luego de catorce años, la estructura disciplinar y administrativa de gran parte de las Universidades ${ }^{3}$ y de las facultades de Educación no ha logrado, ni se ha interesado por explorar la inclusión de esta problemática en el horizonte formativo de los maestros cuya área disciplinar no forma parte de las ciencias naturales y afines. La tarea no es fácil, pues la relación con la naturaleza, incluso pensada desde lo ambiental, no se resuelve con adiciones superfluas de contenidos

2 Ratificada mediante la Ley 1549 de 2012.

3 Salvo algunas dependencias de la Universidad Nacional, como el Instituto de Estudios Ambientales. 
curriculares, sino que implica un proceso de resignificación y reforma radical tanto de las instituciones, como de las concepciones y las prácticas en cuanto a la producción de conocimiento y frente a la vida misma, como se explica más adelante.

Ahora bien, en aras de delimitar las múltiples posibilidades asociadas a la formación sobre la relación entre el ser humano y la naturaleza, en nuestra propuesta optamos por enfatizar en la dimensión estética, pues favorece el "rechazo del modelo de crecimiento ilimitado, de la mercantilización de los afectos y sentimientos, de la ética y de la estética" (Tiezzi, 2006, pp. 77-78). En tal sentido, el acento en la dimensión estética responde, por un lado, a los desafíos que esta conlleva para la formación de la subjetividad y, por otro, a su lugar estratégico para afrontar las técnicas de gobierno en la actualidad. Según Lazzarato, en contraste con lo que predomina en la modernidad, ahora

[...] ya no opera primariamente el castigo o el adiestramiento del cuerpo, sino la modulación de los afectos, sensaciones y deseo [...] el gobierno sobre los hombres no se concentra en instituciones disciplinares orientadas a producir y reproducir la fuerza de trabajo, sencillamente porque en el posfordismo no se trata ya de producir a los productores, sino de producir a los consumidores. (Castro-Gómez, 2009, p. 29)

De allí que los sentidos, la sensibilidad, la emotividad y la afectividad sean considerados aspectos indispensables para que los seres humanos y sociedades descifren y enfrenten los desafíos de nuestro tiempo (Tiezzi, 2006; De Sousa, 2012).

\section{Lo disciplinar, lo interdisciplinar y lo transdisciplinar}

El segundo eje correspondió al desafío y la oportunidad que implicaba emprender una aventura más allá de los límites disciplinares. En consonancia con el proyecto formativo de la Licenciatura, nuestra propuesta de práctica profesional aportó elementos para "fomentar y desarrollar una cultura de la interdisciplinariedad-transdisciplinariedad" (Licenciatura en Educación Básica con énfasis en Humanidades, Lengua Castellana, 2013, p. 13). En efecto, la interdisciplinariedad y la transdisciplinariedad ofrecen alternativas para superar la compartimentación entre las disciplinas; que se concreta en varios ámbitos: epistemológicos, sociológicos, institucionales y, en su forma más extrema, legales (Uribe y Núñez, 2013, p. 27). Sin embargo, no basta con describir o cuestionar las fronteras entre las disciplinas. Es crucial, sobre todo, explorar su capacidad y posibilidades de relacionamiento entre sí y con saberes y conocimientos no académicos, ni disciplinares. Según esto, la interdisciplinariedad y la transdisciplinariedad no son homogéneas, ni fórmulas premeditadas. Por eso se asumieron como un desafío que se configura en medio de los alcances, dificultades, limitaciones y oportunidades emergentes del cruce entre disciplinas como la semiótica, la estética, la pedagogía, la educación ambiental o la permacultura, pero, además, y no menos importante, a partir de la tensión con saberes invisibilizados y menospreciados, frecuentemente, por la academia en contextos formativos escolares y no escolares (por ejemplo, saberes indígenas, afro y populares).

El desafío de interpelar la estructura disciplinar que predomina en el ámbito de la formación de maestros de Humanidades y Lengua Castellana significa apostar por la emergencia de miradas no dicotómicas, ni ahistóricas. Lo cual no implica des-disciplinar, ni des-instrumentalizar la Facultad por completo, sino más bien "que las lógicas disciplinarias e instrumentales puedan coexistir con otras no disciplinarias y no instrumentales dentro de la misma institución" (Castro-Gómez, 2013, p. 43). Por ello, la apuesta epistémica de la propuesta valoró críticamente los aportes disciplinares provenientes del campo de la formación en lenguaje, como los enfoques semiótico-discursivos y socioculturales de la literatura y de los otros sistemas simbólicos (pensamientos y expresiones no alfabéticas, cine, teatro, juegos tradicionales, juegos de mesa, cómic, música, manualidades y medios 
masivos de comunicación). Igualmente, se reconocieron las limitaciones e insuficiencias de estos aportes disciplinares al momento de comprender diversas problemáticas vinculadas con los procesos formativos escolares y no escolares sobre la relación del ser humano con la naturaleza.

Por otro lado, asumimos la revisión crítica de los lenguajes y las formas disciplinares como un proceso de descolonización que no debe quedarse en el orden de la abstracción e intangibilidad vinculada únicamente a la teorización, ni a la toma de conciencia de la herencia colonial. Por eso, suscribimos la crítica y apuesta de Rivera Cusicanqui cuando plantea que "la descolonización no puede ser solo un pensamiento o una retórica, porque las palabras suelen desentenderse de las prácticas" (2010, p. 6). De allí el interés por el lenguaje vinculado a las prácticas y a la ampliación de sentidos como posibilidad de resignificar alternativas de vida, bienestar y desarrollo. Esta cuestión es crucial, porque la crisis de las humanidades está en relación directa con problemáticas humanas y sociales contemporáneas y cotidianas de gran calado. Por lo mismo, no podemos extraviarnos, ni engolosinarnos en una redundante producción de diagnósticos y críticas teóricas. En la Licenciatura hemos corroborado que la discusión teórica sobre la crisis de las humanidades solo es relevante si se sitúa en el ámbito de las prácticas pedagógicas. Desde allí seguimos a Rivera Cusicanqui (2010), quien privilegia las prácticas decolonizadoras en lugar de los discursos o teorías decoloniales.

En el caso concreto de la propuesta de práctica profesional esto se reflejó, por ejemplo, en el interés por los materiales y las mediaciones didácticas. Lo cual permitió visibilizar y problematizar la voz del maestro desde su creatividad y quehacer, en lugar de valorarla únicamente por cualidades intelectuales y capacidades de abstracción escindidas del ámbito contingente de las prácticas. Esto nos situó en otra de las tensiones contemporáneas que esboza Saldarriaga (2003): unos reivindican la figura del maestro como intelectual de la pedagogía y otros como artista creativo, pero "¿Cuál es el umbral mínimo aceptable entre la mecanicidad (automatismo) del método y la capacidad de innovación del maestro?" (p. 144). Tal interrogante lo asumimos en el diálogo inacabado que emprendimos con la educación ambiental,

Educar en lo ambiental es reconfigurar la sensibilidad [...]. Menos metodología y más un atreverse a caminar por las sendas del sentimiento-pensamiento ambiental. No creación de empresa ambiental y sí creación de un sentimiento-pensamiento ambiental, lo que solo es posible deviniendo habitantes-artistas, habitantes-poetas. Recordemos que poético viene de póiesis y póiesis es crear, construir, hacer. Entonces recomendamos que el maestro devenga poeta. (Noguera, 2013, p. 22)

Más allá del cliché, el maestro poeta (creador-artesano-hacedor) lo exploramos mediante discusiones conceptuales, anécdotas y diversas experiencias de la práctica profesional orientadas al análisis, el diseño, la implementación y la configuración-construcción colaborativa de mediaciones y materiales didácticos, para propiciar nuevas percepciones y comprensiones de la relación entre ser el humano y la naturaleza frente a las miradas predominantes y a partir de elementos disciplinares, interdisciplinares y transdisciplinares relevantes para la formación en lenguaje en contextos alternativos o no escolares ${ }^{4}$.

\section{Formación y conocimiento en contextos no escolares}

El tercer eje emergente fue la formación de maestros en contextos alternativos o no escolares. Contemplar el desarrollo de prácticas profesionales en espacios diferentes al aula regular de clase fue un logro de la transformación curricular de la Licenciatura en 2013. La práctica profesional en escenarios alternativos alude a "procesos en los cuales maestras y maestros de humanidades construyen conocimiento por medio de la investigación

4 Para saber detalles, véanse los trabajos de grado resultantes de la propuesta y referenciados en la nota 1. 
en espacios, lugares y contextos distintos al tradicional aula de clase de la escuela regular" (Licenciatura en Educación Básica con énfasis en Humanidades, Lengua Castellana, 2013, p. 25).

En nuestra propuesta, la Corporación Ambiental GEDI de Itagüí, Antioquia, fue el contexto alternativo en el que se llevaron a cabo las prácticas profesionales de los maestros en formación. Mediante diversos proyectos auspiciados por entidades estatales y ONG, las apuestas de GEDI se remiten a la educación y cultura ambiental; intervenciones ambientales; agroecología, seguridad alimentaria y nutricional; áreas naturales protegidas y ecosistemas naturales estratégicos; patrimonio ambiental y liderazgo juvenil ambiental. Recientemente, diversas actividades y programas han empezado a incorporar saberes tradicionales de comunidades campesinas e indígenas, mediante la invitación y participación de integrantes de estos pueblos o bien por la apropiación vivencial que han hecho los miembros de GEDI de tales saberes. De igual modo, un ámbito central de interés de GEDI, que se ha consolidado en los últimos años, ha sido la formación de jóvenes en contextos urbanos y mediante herramientas estéticas.

Desde la Facultad de Educación, el campo de acción de GEDI nos ha ubicado frente al desafío de reconocer y aprender a dialogar con lugares no académicos en los que se producen experiencias y conocimientos sobre la formación de los seres humanos. En efecto, en el paradigma de la modernidad se validan y legitiman ciertas formas y lugares en la producción de conocimiento, mientras que se menosprecian o desconocen otras. En la transición hacia otros paradigmas no debe limitarse la producción de conocimiento relevante a lugares académicos institucionales. Este es un reto para las ciencias sociales y las humanidades que implica reconocer que los actores también pueden tener el lugar de autores de comprensiones y salidas a diversas problemáticas sociales. Una manera en que asumimos esto fue mediante la participación activa, decisiva y continua de dos integrantes de
$\mathrm{GEDI}^{5}$ en la autoría de esta propuesta de práctica profesional, así como en la planeación y desarroIlo de las sesiones en la Universidad y en GEDI.

\section{Otras cuestiones emergentes}

Resignificar lo ambiental implicó explorar posibilidades concretas y locales de desescolarizar (descolonizar) los sentidos y las maneras vinculadas con la dimensión estética de la formación de los seres humanos en su relación con la naturaleza y con su vida en particular. En efecto,

[...] una pedagogía de lo ambiental es una pedagogía en la piel. Pedagogía del sentir, del sentimiento, de la sensación, de la vida. Comprender el alfabeto de la naturaleza en su singularidad es posible si y solo si abandonamos el sujeto y nos disolvemos en la naturaleza. (Noguera, 2004, p. 17)

Para percibir y asumir la disolución del sujeto en la naturaleza, cualquier reflexión epistémica o herramienta conceptual disciplinar moderna resultan insuficientes. En aras de ejemplificar esto en diversas sesiones de trabajo, nos hemos remitido a los hijos del tabaco, la coca y la yuca dulce ${ }^{6}$. Para ellos, disolverse en la naturaleza implica reconocer, experimentar y usar el desvanecimiento de las fronteras entre el humano, la planta y el animal; así como también entre el sentimiento, la palabra y la acción; entre el adentro y el afuera; lo inmaterial y lo material, incluso entre el pasado y el presente. Las características sensibles y materiales de esas fronteras e intercambios entre lo humano y lo no humano permiten entender por qué, para esta comunidad indígena, la formación de los seres humanos se centra en el cuerpo y en la necesidad de

5 Nos referimos Paula Andrea Urrego (pauaurrego@gmail.com, corpogedi@gmail.com), licenciada en Educación Básica con énfasis en Ciencias Naturales, y a Julián Esteban Galvis (galvis.je@ gmail.com), estudiante de la Licenciatura en Educación Básica con énfasis en Ciencias Naturales.

6 Esta es la manera como se autodenomina un conjunto de pueblos la Amazonía colombo-peruana. Su organización ceremonial tiene como eje el uso del tabaco, la coca y la yuca dulce (Candre-Kinerai y Echeverri, 2008; Parra, 2016). 
conocer y cuidar sus relaciones con los alimentos (que forman el cuerpo).

Esto se entiende mejor mediante la explicación que hace Echeverri de un canto-oración del abuelo Kinerait. Cuando este habla de biko (casa), "se refiere al cuerpo, a la maloca o al firmamento, o a los tres simultáneamente" (Candre-Kinerał y Echeverri, 2008, p. 213). Así, experimentar y conocer la conexión entre las tres casas (biko) significa volver a un caos indiferenciado, para luego reordenar y diferenciar esos tres campos o cuerpos: la realidad personal, la social y la natural. De manera general, esta es la base de lo que se enseña y aprende en los consejos, en los cantos-adivinanzas y en otras actividades rituales de los hijos del tabaco, la coca y la yuca dulce (Parra, 2016).

Aprender de los indígenas en el contexto de una Licenciatura en Humanidades, Lengua Castellana detonó múltiples desafíos desde la tensión entre lo indígena y lo no indígena en nuestra propuesta de práctica profesional. En principio, reconocimos que cualquier aproximación a lo indígena que pretenda distanciarse de miradas sociocéntricas y eurocéntricas, precisa de un encuadre histórico y biográfico que favorezca asumir la diferencia cultural como diferencia colonial (Castro-Gómez, 2005). Solo así es posible tomar conciencia y alterar el principio dicotómico y maniqueo que niega e impide cualquier intento de intercomprensión entre lo indígena y lo no indígena, el dialecto bue y el castellano, la selva y la ciudad. De hecho, confirmamos que el mayor reto de las ciencias sociales y las humanidades "no es ya distanciarse de las otras epistemes -con el propósito de garantizar una mayor objetividad del conocimiento- sino acercarse a ellas" (Castro-Gómez, Schiwy y Walsh, 2002, p. 12). En nuestro caso, el distanciamiento consiste en sentidos dicotómicos que sugieren, por ejemplo, que lo indígena es subdesarrollado y lo blanco desarrollado, o bien, que lo indígena es bueno y lo no indígena es malo, o viceversa. No obstante, gracias a los sentidos y actividades generadas durante el proceso fue posible, por momentos, tomar conciencia de las distinciones y dicotomías que separan, así como reconocimos un rasgo que, en lugar de separar, comunica y favorece la interrelación e intercomprensión entre lo indígena y lo no indígena. Se trata de entender la humanidad como humanización, es decir, no como una esencia dada, sino como un proceso que solo es posible en relación con la vida (naturaleza) y su diversidad. Conviene recordar el reiterativo consejo del abuelo Alfonso al respecto, "aquí no hay distinciones. Solamente en hablar diferentes palabras, pero es lo mismo. No hay otra gente, somos los mismos, sea gringos, la misma sangre tenemos" (Parra, 2016, p. 271).

La exploración de los saberes indígenas de esta comunidad para resignificar lo ambiental y nuestra relación con la naturaleza implicó, por un lado, reevaluar "el rol de la intuición, del imaginario, de la sensibilidad y del cuerpo en la transmisión de los conocimientos" (Anes et al., 1994) y, por otro, resignificar nuestra relación con las categorías y las formas del conocimiento moderno y con otras maneras de conocimiento. Por eso, precisamente, además de los aportes de la ecología y otras ciencias, decidimos explorar también los aportes de los hijos del tabaco, la coca y la yuca dulce, a través de materiales bibliográficos, audiovisuales y discusiones. Otra motivación residió en lo afirmado durante el Primer Congreso Nacional Indígena de 1982, mucho antes del auge decolonial en la academia:

El bagaje cultural de las comunidades indígenas tiene un gran valor estratégico en el proyecto de reconstrucción de una nueva sociedad y cultura: sus patrones tradicionales alimenticios, médicos, educativos, agrícolas, políticos, etc., son fundamentales para la investigación de modelos de vida propios e independientes para todos los colombianos. Los modos de vida de nuestras sociedades tradicionales, aun la de las actuales, están llenas de enseñanzas que serán útiles para todo el pueblo en la sociedad futura que vamos a construir. (ONIC, 1982, p. 212)

Explorar y experimentar un diálogo con saberes de los hijos del tabaco, de la coca y de la yuca dulce 
para resignificar lo ambiental, favoreció leer(nos) y sumergirnos en la crisis de las humanidades, desde un ámbito local, concreto e incierto para la formación de maestros de lengua y literatura, que no se distrae en la defensa o ataque desgastante de currículos literarios, valores humanos eurocéntricos, ni otras prácticas que, por lo general, edulcoran o invisibilizan la herencia colonial y colonizadora de las humanidades modernas. También hemos reconocido que resultaría improductivo conformarnos con hacer de esta constatación una denuncia y diagnóstico interminable que estanque la posibilidad de formación y transformación de nuestra relación con la naturaleza y la vida, dado que

[...] la preocupación fundamental está en el cuidado y el posibilitamiento de la vida, esto es, de la vida humana, pero con ella, al lado de ella, exactamente al mismo nivel y sin otros miramientos, contemporánea con ella, también de la vida en general en el planeta. (Maldonado, 2000, p. 69)

\section{A modo de conclusión}

Explorar la generación de experiencias y situaciones que favorezcan la percepción y comprensión de la interdependencia e interconexión del ser humano y la naturaleza es un desafío que forma parte de los grandes problemas sociales y humanos contemporáneos. Pues,

[...] lo que está en juego no es solo un modelo económico (el capitalismo neoliberal), un conjunto de rasgos culturales hostiles a la vida en el planeta (como el individualismo rampante y el consumismo), las políticas ambientales globales, las luchas geopolíticas de poder para re- y desoccidentalizar el mundo, ni el complejo militar-industrial. Todos estos están involucrados, sin duda, pero para quienes la coyuntura actual lo "cambia todo", inicialmente hay que cambiar es toda una forma de vida y todo un estilo de creación de mundos. (Escobar, 2016, p. 11)

Frente a tal desafío, consideramos que los cuatro ejes derivados de la propuesta de práctica profesional pueden funcionar como alternativas, pues ofrecen elementos para cuestionar y resignificar aspectos epistémicos, conceptuales y metodológicos, en aras de continuar la exploración derivada de nuestra propuesta en diversos escenarios. De hecho, los espacios de discusión y visibilización de nuestra experiencia se han dado en las reuniones de trabajo del grupo de profesores-asesores de la Licenciatura, en eventos académicos donde los estudiantes socializaron sus trabajos y, finalmente, los cuatro ejes perfilados han permitido que la Licenciatura le dé continuidad a esta propuesta de práctica profesional con nuevos estudiantes y durante tres semestres (2017-Il y 2018); lo cual ha permitido fortalecer la alianza y construcción colaborativa con GEDI.

Reiteramos que los cuatro ejes esbozados son los aprendizajes que emergieron del desafío que representó arriesgarnos a cuestionar y trastocar los senderos trazados por los saberes y los propósitos disciplinares sobre los cuáles se fundamenta la Licenciatura, así como subvertir algunas de las maneras oficiales de transitarlos y nombrarlos en diálogo con saberes, propósitos y problemáticas por fuera (o en los bordes) del horizonte de la lingüística, la literatura, la didáctica y las humanidades modernas en un contexto como el de GEDI, donde no hay currículo, ni calificaciones, tampoco es obligatorio asistir ni permanecer; mucho menos se considera que para que se produzca el aprendizaje es necesario agrupar (separar) a las personas por edades.

Un desafío adicional es entender que indisciplinar la formación en lenguaje, como se pretendió desde el título de la propuesta de práctica profesional, conlleva indisciplinar la formación en investigación educativa ${ }^{7}$. Pese a la apuesta de la Licenciatura por una "formación de maestros lectores y transformadores de contextos a partir de relaciones inter- y transdisciplinares desde los saberes socioculturales pedagógicos y didácticos" (Licenciatura en Educación Básica con énfasis en

7 La apuesta por indisciplinar hace eco de la propuesta de Castro-Gómez, Schiwy y Walsh (2002). 
Humanidades, Lengua Castellana, 2013, p. 5), hemos constatado que prevalece, salvo contadas excepciones ${ }^{8}$, una investigación y una relación con el saber disciplinar donde escasean posibilidades, recursos, lenguajes, mecanismos de validación y enfoques investigativos que no sean prestados (casi siempre caricaturizados) de las ciencias sociales y de las ciencias exactas o experimentales ${ }^{9}$.

Lo anterior se manifestaba en ciertas inquietudes de los estudiantes durante el primer semestre (2016-I). Debido a sus experiencias en cursos anteriores y a sus conversaciones con compañeros que cursaban también las prácticas profesionales en otros grupos, nuestros estudiantes se preguntaban si sus temáticas y, por extensión, sus investigaciones tendrían alguna relevancia, seriedad y pertinencia para los propósitos y saberes de una Licenciatura en Humanidades, Lengua Castellana; además se preguntaban, ¿cómo los evaluarían los jurados de sus trabajos de grado y sus compañeros? Tales inquietudes fueron abordadas en varias sesiones de trabajo y en sus respectivas investigaciones, pues remitían a una cuestión central: el extrañamiento de su perfil o identidad profesional.

Después de todo, la disciplina ofrece una identidad profesional, un reconocimiento púbico, una experticia recompensada institucionalmente. Aventurarse a vivir sobre los puentes [interdisciplinarios o aproximaciones transdisciplinarias] se toma como una pérdida, incluso como carencia y como falta de rigurosidad y cientificidad. (Castro-Gómez, 2013, p. 39)

Es por ello que consideramos que esta primera experiencia de práctica profesional inició el tránsito por procesos de desescolarización, desinstitucionalización y desaprendizaje de la modernidad y del desarrollo, en medio, y en relación, con sus saberes e instituciones insignes (las humanidades, una licenciatura y el dispositivo escolar). Dimos los primeros pasos en este proceso desde el

8 Se trata de una línea de práctica profesional sobre investigación narrativa.

9 Como, por ejemplo, la investigación basada en artes. ámbito local de las prácticas, los cuerpos, la palabra y nuestras biografías. Estos son los lugares más productivos y fértiles para renovar una manera de pensar, hablar y caminar la formación en lenguaje más allá del corsé escolar, moderno y colonial. Ese que nos anestesia, que raras veces percibimos y, menos aún, logramos recordar cómo se tejió y cómo tejemos cotidianamente.

En otras palabras, seguimos aprendiendo cómo la transformación en la manera de pensar y llevar a cabo la formación en lenguaje en relación con la naturaleza, desde una dimensión estética, precisa de un ejercicio complejo de descolonización del lenguaje académico y no académico para ampliar las maneras de hablar, escuchar y hacer. En consonancia con lo planteado por Rivera Cusicanqui: "La posibilidad de una reforma cultural profunda en nuestra sociedad depende de la descolonización de nuestros gestos, de nuestros actos, y de la lengua con que nombramos el mundo" (2010, pp. 70-71).

Así, explorar una resignificación de lo ambiental implica explorar unas humanidades que reconocen, interpelan y buscan distanciarse de su arraigado antropocentrismo y de la separación entre cultura y naturaleza. Esta es una tarea (trans) formativa de largo aliento, pues implica

[...] aprender que los valores no son ni exclusiva ni prioritariamente humanos. Por el contrario, la preocupación por los valores se revela, en verdad, como la preocupación por valores no antropocéntricos -dentro de los cuales quedan incluidos los valores propios de cualquier tipo de humanismo-. (Maldonado, 2000, p. 72)

Durante la práctica profesional, esto significó convivir con la extrañeza y la incertidumbre que conlleva pensar y propiciar procesos investigativos y formativos en el área del lenguaje por fuera (aunque no en contra) del horizonte delimitado por las disciplinas. Extrañeza e incertidumbre que se experimentó no solo como un conflicto cognitivo, sino, sobre todo, ético, afectivo y visceral, tanto para los estudiantes como para quienes acompañamos 
su proceso. Es por ello que resignificar la relación con la naturaleza es una oportunidad y un proceso formativo que no se resuelve en un semestre universitario, ni en dos; tampoco es suficiente la adición de lecturas y materiales bibliográficos al pénsum tradicional, pues lo que conlleva lo ambiental son cuestiones alojadas en nuestra manera de ver la vida y vivirla, personal, familiar y socialmente. Por lo mismo, no es un problema de acceder a información actualizada sobre educación ambiental que se articula con temas literarios o con la enseñanza de la lectura y la escritura. En lugar de ello, el proceso consiste en arrojarnos a la extrañeza del desafío creativo-formativo que implica des-atrofiar y des-anestesiar nuestra capacidad de sentir y percibir la vida y, por ende, de renovar las posibilidades de nombrarla y valorarla, en una época donde las dinámicas del mercado invaden, con apetito insaciable, cada lugar y cada tiempo del cuerpo, del pensamiento, del sentir, del quehacer, de las comunidades y del territorio.

\section{Referencias bibliográficas}

Anes, J., et al. (noviembre de 1994). Carta de la Transdisciplinariedad. Recuperado de http://www.filosofia.org/cod/c1994tra.htm

Candre-Kinerai H. y Echeverri, J. (2008). Tabaco frío, coca dulce. Leticia: Universidad Nacional.

Capra, F. (2003). La trama de la vida. Una nueva perspectiva de los sistemas vivos. Barcelona: Anagrama.

Castro-Gómez, S. (2000). Ciencias sociales, violencia epistémica y el problema de la "invención del otro". Recuperado de http://bibliotecavirtual.clacso.org.ar/ar/libros/lander/castro.rtf

Castro-Gómez, S. (2005). La hybris del punto cero: ciencia, raza e ilustración en la Nueva Granada (17501816). Bogotá: Editorial Pontificia Universidad Javeriana.

Castro-Gómez, S. (2009). Noopolítica y sociedades de control: las subjetividades contemporáneas en Mauricio Lazzarato. En J. Martínez y F. Neira (comp.). Miradas sobre la subjetividad (pp. 21-38). Bogotá: Universidad de la Salle

Castro-Gómez, S. (2013). Desafíos de la inter- y la transdisciplinariedad para la Universidad en Colombia. Trans-pasando Fronteras, 3, 33-45. https://doi. org/10.18046/retf.i3.1625

Castro-Gómez, S. (noviembre, 2014). Descolonizar las artes. Una genealogía del modelo de la universidad-empresa en Colombia. Conferencia Doctorado Interinstitucional en Educación. Universidad Distrital Francisco José de Caldas. Recuperado de http://udistrital.edu.co:8080/c/document_library/ get_file?uuid=ae03941c-17e6-4a83-a246-9c0b11 ea4753\&groupld $=98864$

Castro-Gómez, S.; Schiwy, F. y Walsh, C. (eds.) (2002). Indisciplinar las ciencias sociales. Geopolíticas del conocimiento y colonialidad del poder. Perspectivas desde lo andino. Quito: Universidad Andina.

Chartier, A.M. (2004). Enseñar a leer y a escribir: una aproximación histórica. Ciudad de México: Fondo de Cultura Económica.

De Sousa, B. (2009). Una epistemología del sur. Buenos Aires: Clacso-Siglo XXI.

De Sousa, B. (2012). De la mano de Alicia: lo social y lo político en la posmodernidad. Bogotá: Siglo de Hombre.

Escobar, A. (2016). Autonomía y diseño. La realización de lo comunal. Popayán: Universidad del Cauca.

Grosfoguel, R. (2006). Actualidad del pensamiento de Cesaire: redefinición del sistema-mundo y producción de utopía desde la diferencia colonial. En: A. Cesaire. Discurso sobre el colonialismo (pp. 147172). Madrid: Akal.

Licenciatura en Educación Básica con énfasis en Humanidades, Lengua Castellana (2013). Proyecto de Formación. Medellín: Facultad de Educación, Universidad de Antioquia [Archivo físico del proceso de autoevaluación].

Licenciatura en Educación Básica con énfasis en Humanidades, Lengua Castellana (2016). Convocatoria Prácticas Pedagógicas. Medellín: Facultad de Educación, Universidad de Antioquia. Recuperado de https://goo.gl/Qe1s7E 
Maldonado, C. (2000). Ideas acerca de la historia y el futuro de las relaciones entre ciencia y filosofía. Recuperado de http://carlosmaldonado.org/articulos/ BEyC\%20Cap.\%202.pdf

Ministerio del Medio Ambiente-Ministerio de Educación (2002). Política Nacional de Educación Ambiental. Bogotá: República de Colombia.

Morín, E. (2011). La vía para el futuro de la humanidad. Paidós: Barcelona.

Noguera, P. (2013). Pensamiento ambiental y arte. Decisio, 34, 17-23.

Nussbaum, M. (2010). Sin fines de lucro. Por qué la democracia necesita de las humanidades. Buenos Aires: Katz Editores.

Ordine, N. (2013). La utilidad de lo inútil. Manifiesto. Barcelona: Acantilado.

Organización Nacional Indígena de Colombia (ONIC) (1982). Conclusiones del Primer Congreso Indígena Nacional. En E. Sánchez y H. Molina (comp.) (2010). Documentos para la historia del movimiento indígena colombiano contemporáneo (pp. 195242). Bogotá: Ministerio de Cultura.

Parra, C. (2016). Hacia la intercomprensión entre lo murui-uitoto y lo no murui-uitoto en procesos formativos sobre la relación con la naturaleza. (Tesis de doctorado). Pontificia Universidad Javeriana, Bogotá.

Parra, C. (2016a). Programa de curso del seminario de Práctica Pedagógica I. (Documento aprobado por el Comité de Carrera de la Licenciatura en Educación básica con Énfasis en Humanidades, Lengua Castellana. Facultad de Educación. Universidad de Antioquia).

Rivera Cusicanqui, S. (2010). Ch'ixinakax utxiwa. Una reflexión sobre prácticas y discursos descolonizadores. Buenos Aires: Tinta Limón.

Saldarriaga, O. (2003). Del oficio de maestro. Prácticas y teorías de la pedagogía moderna en Colombia. Bogotá: Magisterio.

Tiezzi, E. (2006). La belleza y la ciencia. Hacia una visión integradora de la naturaleza. Barcelona: Icaria.

Uribe, C. y Núñez, J. (2013). Interdisciplinariedad y transdisciplinariedad: ¿colaboración o superación de las disciplinas? En C. Uribe (ed.). La interdisciplinariedad en la Universidad Contemporánea (pp. 26-63). Bogotá: Editorial Pontificia Universidad Javeriana.

\section{(c) (1) $\Theta \Theta$}

